\title{
ORIGINAL RESEARCH \\ Nasal Cavity Eosinophilic Angiocentric Fibrosis: CT and MR Imaging Findings
}

B.T. Yang

Y.Z. Wang

X.Y. Wang

Z.C. Wang

BACKGROUND AND PURPOSE: EAF consists of exceedingly rare lesions and presently lacks specific imaging reports. The purpose of this study was to determine the CT and MR imaging features of nasal cavity EAF.

MATERIALS AND METHODS: We retrospectively reviewed 6 patients with histology-proven EAF in the nasal cavity. All 6 patients underwent CT and 3 patients also underwent MR imaging. The following imaging features were reviewed: 1) location, 2) shape, 3) margin, 4) CT attenuation, and 5) MR imaging signal intensity. In addition, the accompanying changes and the TIC of the DCE MR imaging were analyzed.

RESULTS: All occurrences of EAF were located at the anterior nasal cavity, arising from the nasal septum in 4 patients, with irregular shape, and the lateral nasal wall in 2, with oval configuration. The mean size was $29.8 \mathrm{~mm}$ (range 12-52 mm). On nonenhanced CT, EAF lesions appeared isoattenuated relative to gray matter. On the T1-weighted image, the lesions were isointense in $3(100 \%)$ patients. On the T2-weighted image, the lesions were isointense in $1(33.3 \%)$ patient and hypointense in 2 (66.7\%) patients. Occurrences of EAF revealed moderate inhomogeneous contrast enhancement. Three patients underwent DCE MR imaging and the TICs exhibited a rapidly enhancing and slow washout pattern. One patient had bilateral lacrimal gland enlargement, which displayed low signal intensity on the T2-weighted image.

CONCLUSIONS: A familiarity with imaging findings, including lesion location, isoattenuation and bony changes, and hypointensity on T2-weighted images may help to accurately diagnose this rare entity.

ABBREVIATIONS: DCE = dynamic contrast-enhanced; EAF = eosinophilic angiocentric fibrosis; ESS = endoscopic sinus surgery; FSPGR = fast-spoiled gradient-recalled echo; $\mathrm{HU}=$ Hounsfield Unit; NK = natural killer; TIC = time-intensity curve; WG = Wegener granulomatosis

E AF is an extremely rare, slowly progressive fibroinflammatory disorder with a predilection for the nasal cavity and, occasionally, the paranasal sinuses, larynx, and orbit. ${ }^{1-20}$ To the best of our knowledge, only 40 cases of this entity have been reported in the English literature since the entity was first proposed by Roberts and McCann in $1985^{1}$; unfortunately, no specific imaging study on this entity was found until now. We reviewed, retrospectively, the CT and MR imaging findings of 6 patients with EAF confirmed by histology. The features in this paper may help to increase the awareness of this entity among both clinicians and radiologists. A prompt diagnosis of EAF will help to facilitate the most appropriate management planning.

\section{Materials and Methods}

\section{Patients}

This study was approved by the institutional review board of Beijing Tongren Hospital. We retrospectively reviewed the CT and MR imaging findings of 6 patients with histology-proven EAF over a 6-year period (March 2004 to June 2010); the 6 patients were selected by review of clinical records. The ratio of female to male was $1: 1$. The average age was 38 years (range $16-73$ years). All 6 patients under-

Received February 11, 2011; accepted after revision March 27.

From the Department of Radiology, Beijing Tongren Hospital, Capital Medical University, Beijing, China

Please address correspondence to Ben Tao Yang, Department of Radiology, Beijing Tongren Hospital, Capital Medical University, No.1, Dongjiaominxiang, Dongcheng District, Beijing 100730, China; e-mail: cjr.yangbentao@vip.163.com

http://dx.doi.org/10.3174/ajnr.A2786 went surgical removal of EAF lesions by ESS. Their clinical medical records were reviewed, and they had no history of allergy, atopy, trauma, or immunologic diseases.

\section{CT Technique}

All 6 patients underwent paranasal sinus unenhanced CT. Images were obtained in both the axial and coronal planes in all 6 patients by using a Lightspeed 16-section CT scanner (GE Healthcare, Beijing, China) or a Brilliance 64-section CT scanner (Philips Healthcare, Best, the Netherlands). The imaging parameters were as follows: voltage, $120 \mathrm{Kv}$; current, $200 \mathrm{~mA}$; matrix, $512 \times 512$; and section thickness, $2 \mathrm{~mm}$. These images were reconstructed by using both a bone algorithm (window width of $2000 \mathrm{HU}$ at a window level of $200 \mathrm{HU}$ ) and a soft-tissue algorithm (window width of $400 \mathrm{HU}$ at a window level of $40 \mathrm{HU}$ ). Reformations were performed from the superior wall of the frontal sinus to the inferior wall of the maxillary sinus on the axial plane, and from the anterior wall of the frontal sinus to the posterior wall of the sphenoid sinus on the coronal plane.

\section{MR Imaging Technique}

Three patients underwent paranasal sinus MR imaging before surgery. The MR examinations were performed on a 1.5T unit MR system (Signa, GE Healthcare, Milwaukee, Wisconsin) with a head coil. Fast spin-echo pulse sequences were used in these patients. These patients underwent pre-enhanced T1WI and T2WI, and postenhanced T1WI, in the axial, coronal, and sagittal planes. The imaging parameters were as follows: T1WI: TR 500-600 ms, TE 10-15 ms; T2WI: TR 3000-3500 ms, TE 120-130 ms; NEX 2-4; echo-train length 11-27; matrix $256 \times 256$; FOV $20 \times 20 \mathrm{~cm}$; section thickness $4-5 \mathrm{~mm}$; intersection gap $0.5 \mathrm{~mm}$. 


\begin{tabular}{|c|c|c|c|c|c|c|c|c|}
\hline \multicolumn{9}{|c|}{ Nasal cavity eosinophilic angiocentric fibrosis: CT and MR imaging characteristics } \\
\hline \multirow{2}{*}{$\begin{array}{l}\text { Patient } \\
\text { No. }\end{array}$} & \multirow{2}{*}{$\begin{array}{l}\text { Sex/ Age } \\
\text { (years) }\end{array}$} & \multirow[b]{2}{*}{ Location } & \multirow{2}{*}{$\begin{array}{l}\text { Lesion Size } \\
(\mathrm{mm})\end{array}$} & \multirow{2}{*}{$\begin{array}{c}\text { Unenhanced } \\
\text { CT }\end{array}$} & \multicolumn{3}{|c|}{ MR Imaging } & \multirow{2}{*}{$\begin{array}{c}\text { Follow-Up } \\
\text { (years) }\end{array}$} \\
\hline & & & & & T1WI & $\mathrm{T} 2 \mathrm{WI}$ & Contrast & \\
\hline 1 & $\mathrm{~F} / 26$ & Nasal septum & 33 & Isodense & Isointense & Hypointense & Moderate & 2 \\
\hline 2 & $\mathrm{M} / 16$ & Nasal septum & 52 & Isodense & Isointense & Hypointense & Moderate & $\mathrm{N}$ \\
\hline 3 & $\mathrm{~F} / 62$ & Right lateral nasal wall & 21 & Isodense & Isointense & Isointense & Moderate & 4 \\
\hline 4 & $\mathrm{M} / 28$ & Nasal septum & 26 & Isodense & $\mathrm{N}$ & $\mathrm{N}$ & $\mathrm{N}$ & 3 \\
\hline 5 & $F / 24$ & Nasal septum & 35 & Isodense & $\mathrm{N}$ & $\mathrm{N}$ & $\mathrm{N}$ & 5 \\
\hline 6 & $\mathrm{M} / 73$ & Left lateral nasal wall & 12 & Isodense & $\mathrm{N}$ & $\mathrm{N}$ & $\mathrm{N}$ & 0.5 \\
\hline
\end{tabular}

Note:- Lesion size indicates maximum diameter. CT density and MR imaging signal intensity are compared with cerebral gray matter. $\mathrm{N}$ indicates none.

Five patients were followed up for $0.5-5$ years following surgery. Patient 1 recurred after 2 years (Fig $3 F$; the other 5 patients showed no evidence of recurrence. After the second surgery, a follow-up of approximately 1 year for patient 1 , there was no clinical evidence of recurrence.

Postcontrast T1-weighted images with frequency-selective fat saturation were obtained in the optimal plane after DCE MR imaging. Rapid manual bolus intravenous injection $(2 \mathrm{~mL} / \mathrm{s})$ of $0.1 \mathrm{mmol}$ of gadopentetate dimeglumine (Magnevist; Bayer Schering, Berlin, Germany) per kilogram of body weight was followed by a $10 \mathrm{~mL}$ flush of normal saline solution. DCE MR imaging was performed by using 3D FSPGR before conventional postcontrast T1WI in 3 patients. The scan parameters were as follows: TR $8.4 \mathrm{~ms}$; TE $4.0 \mathrm{~ms}$; NEX 1; matrix $256 \times 160$; FOV $20 \times 20 \mathrm{~cm}$; section thickness $3.2 \mathrm{~mm}$; and intersection gap $0 \mathrm{~mm}$. A total of 12 sets of dynamic images were acquired. Each set included 6 images and required 13 seconds; the interset time gap was 12 seconds. The whole dynamic series took 5 minutes in total. After the dynamic scan, source images were transferred to an ADW 4.2 workstation (GE Healthcare) for further analysis. In the maximal section of the lesions, the authors manually drew ROIs for signal intensity measurements to avoid cystic and necrotic areas. ROIs were approximately 3-4 $\mathrm{mm}$ in diameter. To ensure the accuracy of the TIC, several ROIs were chosen on the basis of lesion size and subsequently compared with the histopathology findings. At the same time, the change in signal intensity of a similar region of interest placed on the masticator muscle was used for reference.

\section{Image Analysis}

The CT and MR images were reviewed on a PACS system by 3 experienced head-and-neck radiologists (B.T.Y., Y.Z.W., X.Y.W., with 13, 8 , and 5 years of experience, respectively), and the findings were reached by consensus.

In the present study, we adopted the classification scheme of the TIC of DCE MR imaging proposed by Yabuuchi et $\mathrm{al}^{21}: 1$ ) Type I (steady enhancement pattern) appears as a straight or curved line, and enhancement continues over the entire dynamic study; 2) Type II (rapidly enhancing and slow washout pattern) appears as growing enhancement in the early stage and then displays a sharp bend to form a plateau in the middle and later stages; and 3) Type III (rapidly enhancing and rapid washout pattern) appears as growing enhancement during the early stage and then progressively decreases in signal intensity.

\section{Results}

The most common symptoms of EAF are nasal obstruction and anterior nasal swelling (6 patients, 100\%), rhinorrhea (4 patients, 66.7\%), epistaxis (2 patients, 33.3\%), and eyelid swelling and proptosis (1 patient, 16.7\%). Before diagnosis, the duration of the symptoms experienced by the 6 patients averaged 3.5 years (range $1-8$ years).

Histologically, the lesions showed characteristic eosinophilic vasculitis and perivascular fibrosis, with a whorled and

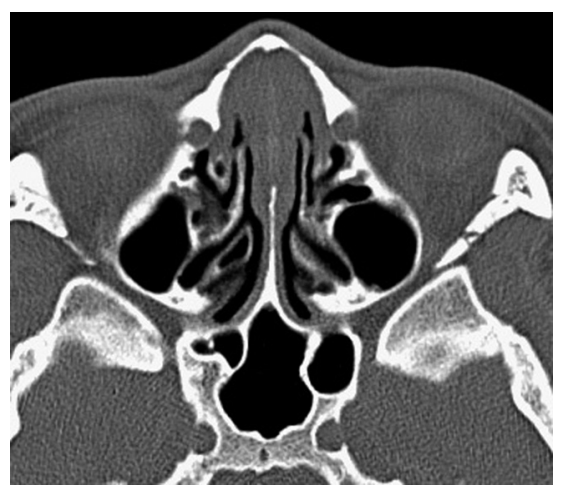

Fig 1. Case 5. Axial CT image shows symmetrical thickening of the septum with lateral nasal walls bilaterally.

onionskin appearance, which proved to be EAF. On immunohistochemical staining, tumor cells were positive for vimentin (4 patients, 66.7\%) and alpha-smooth muscle (2 patients, $33.3 \%)$. The Ki-67 index was approximately $1 \%$ in 3 patients (50\%).

All EAFs originated from the anterior nasal cavity. Four $(66.7 \%)$ lesions occurred in the nasal septum and progressively extended into the lateral nasal wall and nasal base, which demonstrated an irregular shape and had ill-defined margins. Two (33.3\%) other lesions with well-defined margins arose from the lateral nasal wall and involved the nasal septum, appearing as an ovoid configuration. The mean maximum diameter was $29.8 \mathrm{~mm}$ (range 12-52 $\mathrm{mm}$ ). The imaging characteristics are summarized in the Table.

On nonenhanced CT, the lesions appeared homogeneously isoattenuated to gray matter (Figs 1 and 2). Nasal septal perforation was identified in $1(16.7 \%)$ patient. In $2(33.3 \%)$ patients, there was slight bony thinning in the frontal process of the maxilla.

Three (100\%) occurrences of EAF appeared isointense on the T1-weighted image, and the lesions appeared hypointense in $2(66.7 \%)$ patients and isointense in $1(33.3 \%)$ patient on the T2-weighted image (Fig $3 A, B$ and Fig $4 A$ ). These lowsignal-intensity foci on the T2-weighted image appeared to be related to fibrosis, as seen in histologic observations. EAF typically showed moderate heterogeneous enhancement on conventional contrast-enhanced images (Figs $3 C$ and $4 C$ ). Besides the nasal abnormality, patient 2 showed bilateral lacrimal gland enlargement, appearing as low signal intensity on the T2-weighted image (Fig 4B). All 3 patients underwent DCE MR imaging, and the TICs revealed a rapidly enhancing and 

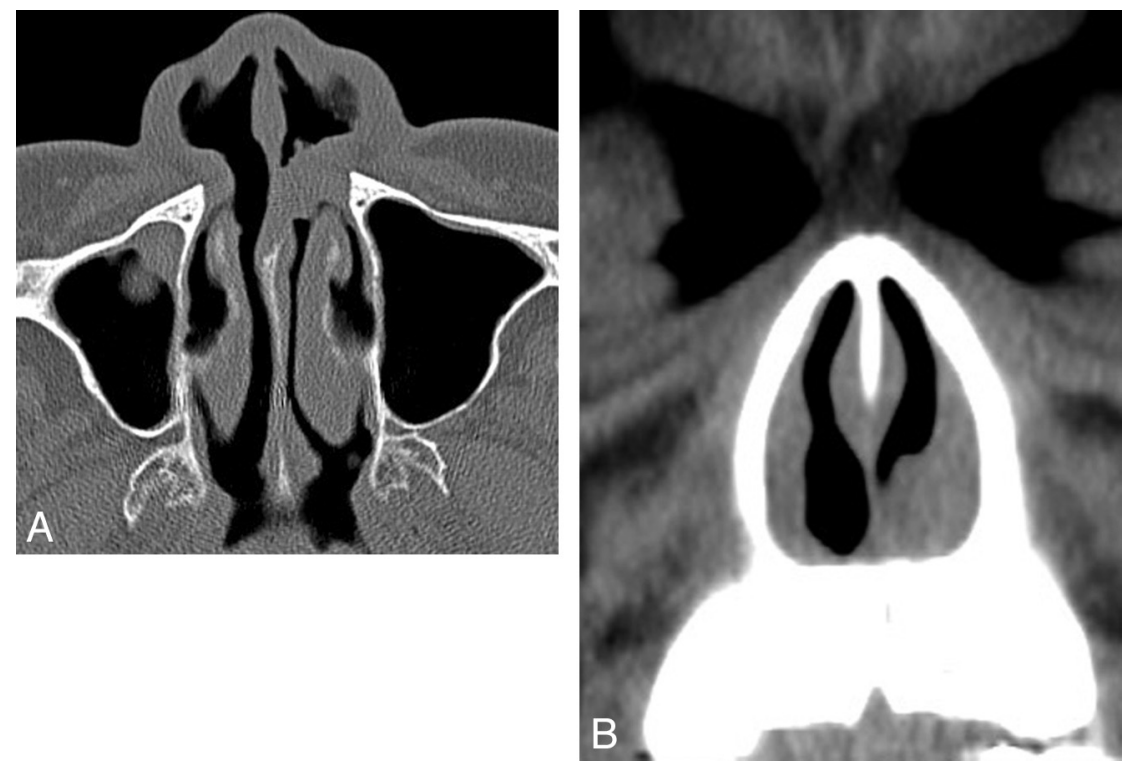

Fig 2. Case 6. Axial $(A)$ and coronal $(B)$ CT images show an oval-shaped, well-defined, and isointense soft-tissue mass arising from left lateral nasal wall and extending into the nasal septum.

slow washout pattern, which were consistent with type II in the classification proposed by Yabuuchi et al ${ }^{21}$ (Fig 3D, E).

\section{Discussion}

EAF is an exceedingly rare benign disease of the nasal cavity, and most of the papers in the literature appear as case reports. This disorder typically demonstrates an unusual submucosal inflammatory, fibrosing, and tumor-like lesion. Currently, the exact etiology of EAF still remains unclear. Allergy, atopy, and trauma have been suggested as predisposing factors, but the evidence for any of these origins is slim..$^{2,4,8,9,11,13,18}$ There was no history of allergy, trauma, previous surgery, self-medication, or known immunologic disease in any of the 6 patients, which does not support the potentially predisposing factors as well.

The typical histologic characteristics are the basis for establishing the diagnosis of EAF. Histologically, this condition generally consists of early and late phases, both of which may be present in a single biopsy, suggesting that the entity is continually evolving. ${ }^{1}$ A vasculitis without fibrinoid necrosis that is predominated by eosinophils is quite characteristic in the early-stage lesion. Perivascular fibrosis with a whorled and onionskin appearance is a characteristic feature in the late phase, at which time eosinophils are almost always present. ${ }^{1,3,5,8,20}$

The clinical features of the 40 patients ( 23 females and 17 males) reported in the literature ${ }^{1-20}$ were pooled with the present 6 patients (total number of patients $=46$ ). There were 26 $(56.5 \%)$ females and $20(44.5 \%)$ males. The average age was 46.7 years (range 16-79 years). The nasal cavity (41/46, $89.1 \%$ ) was by far the most common site, including localized nasal cavity involvement $(29 / 46,63 \%)$ and coexistent adjacent anatomic site disease $(12 / 46,26.1 \%){ }^{1-20} \mathrm{EAF}$ is an indolent, slow-progressing condition that often evolves over many years, and the patients usually describe a worsening of symptoms. Clinical manifestations were nonspecific for our cases as well as for those reported in the literature. ${ }^{1-20}$ Patients with nasal EAF typically presented with progressive nasal obstruction. Rhinorrhea, epistaxis, and facial pain, while not common, were occasionally present. Nonspecific mucosal thickening, submucosal fullness, and crusting were often detected on clinical examination.

Most occurrences of EAF originated from the anterior nasal cavity, with the nasal septum being the most commonly affected location. Only a minority of cases arising from the lateral nasal wall or paranasal sinuses have been found. ${ }^{1-14,19,20}$ According to the literature and the present study, the typical imaging finding is soft-tissue thickening of the septum and lateral nasal walls. ${ }^{2,5,7,8,11-14}$ On nonenhanced CT, EAF usually shows homogeneous isodensity compared with gray matter and rarely has calcification. As the lesions progress, they may cause adjacent bone remodeling, thinning, slight absorption, or even sclerosis. EAF is generally isointense relative to gray matter on T1-weighted images, with moderate inhomogeneous enhancement after contrast administration. Most lesions exhibit hypointense signal intensity on T2-weighted images, owing to fibrosis seen in the late-phase lesions. Low signal intensity on T2-weighted images is an important imaging feature that probably gives a diagnostic clue to this entity in this region; lesions seldom reveal isointense or slight hyperintense signal intensity on T2-weighted images. In our series, patient 2 exhibited bilateral lacrimal gland enlargement, appearing as low signal intensity on T2-weighted images, which apparently is extremely rare, according to our literature search, and could easily be mistaken for WG or sarcoidosis. Characteristic diagnostic features of attenuation and signal intensity can aid in detecting EAF of this region; however, based on the present 6 patients, other identifying characteristics are as follows: 1) long history; 2) nasal septum involvement; 3 ) ill-defined margin; 4) lack of bone destruction; 5) type II (rapidly enhancing and slow washout pattern) TIC.

DCE MR imaging can provide information related to tumor perfusion, microvascular permeability, and volume of the extracellular space, information that may help to diagnose 

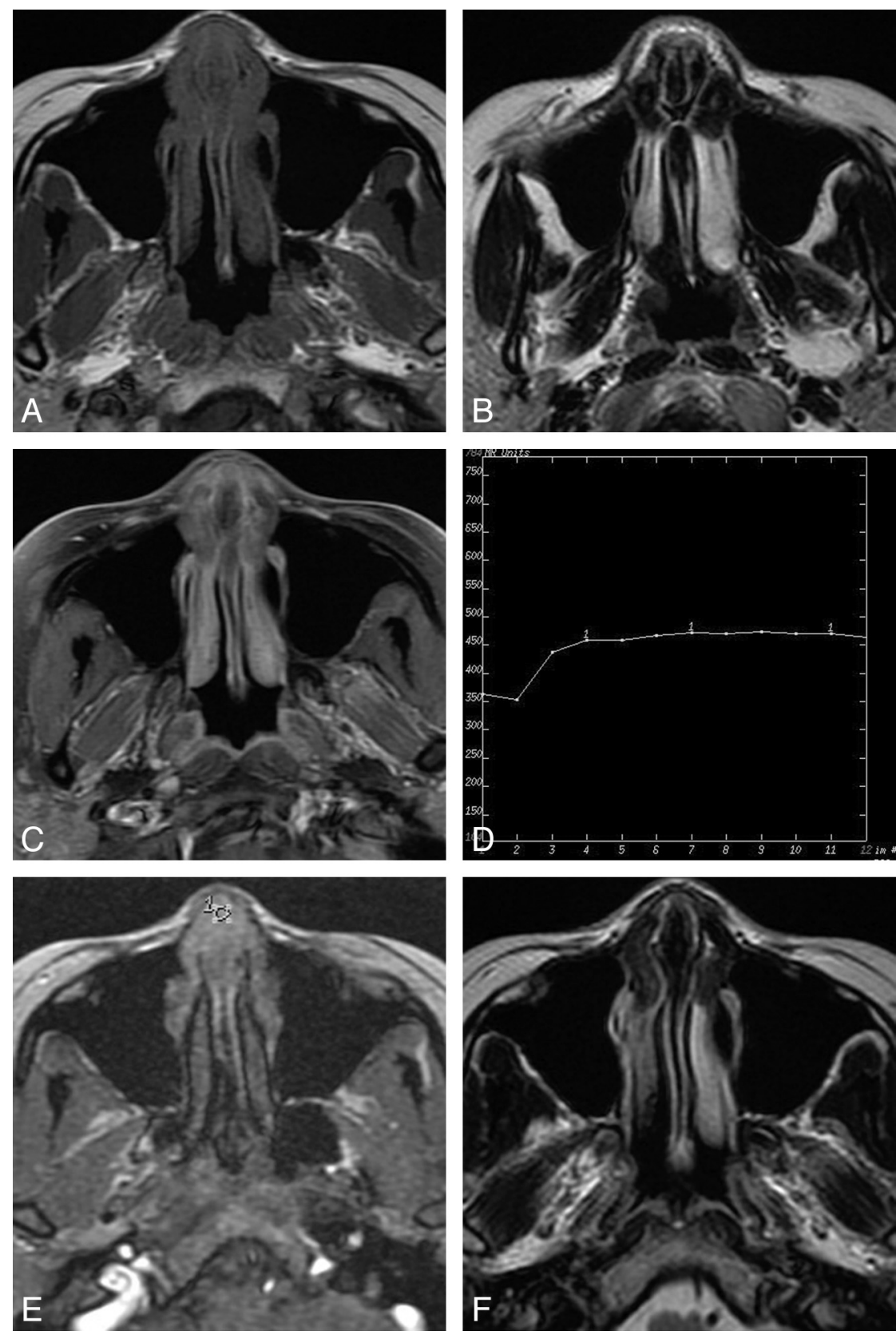

Fig 3. Case 1. $A$, Axial T1-weighted image shows symmetrical thickening of the septum and lateral nasal walls with isointense signal intensity. $B$, The lesion has hypointense signal intensity on the axial T2-weighted image. $C$, Axial contrast-enhanced T1-weighted image with fat saturation shows moderate inhomogeneous enhancement of the lesion. $D$, Corresponding axial DCE MR image depicts the rapidly enhancing and slow washout pattern (Type II). E, The round cursors mark the region of interest selected for signal intensity measurement at dynamic MR imaging. F, Axial T2-weighted image shows a recurrent lesion in the primary site.

some lesions and predict their biologic behavior. ${ }^{21}$ For example, a TIC of the DCE MR imaging may provide valuable information in differentiating benign from malignant lesions. ${ }^{21}$ The TICs of our 3 patients in the present study appeared as rapidly enhancing and slow washout type, which suggests a benign lesion. To the authors' knowledge, there have been no previously documented reports on DCE MR imaging of EAF of the nasal cavity in the literature. This technique may therefore serve as an additional method for predicting the nature of a suspected EAF in the nasal cavity.

Although some imaging features may suggest the diagnosis, they are not specific for EAF and can be seen in other benign lesions in the nasal cavity. The differential diagnosis primarily includes WG, nasal NK/T-cell lymphoma, inverted papilloma, polyps, hemangioma, and schwannoma. WG is a vasculitic process that can involve multiple anatomic sites of the body, including the sinonasal cavity, orbit, temporal bone and other locations in the head and neck. The typical imaging features of WG in the sinonasal cavity include midline destruction similar to postsurgery appearance; bony sclerosis of the affected paranasal sinuses, causing partial or complete obliteration of the sinus cavities; concomitant orbital mass, pterygopalatine fossa, or masticator space infiltration; and serous otitis media. A blood test positive for c-ANCA may support a suspected diagnosis of WG. ${ }^{22}$ Nasal NK/T-cell lymphoma characteristically exhibits soft-tissue thickening of the 

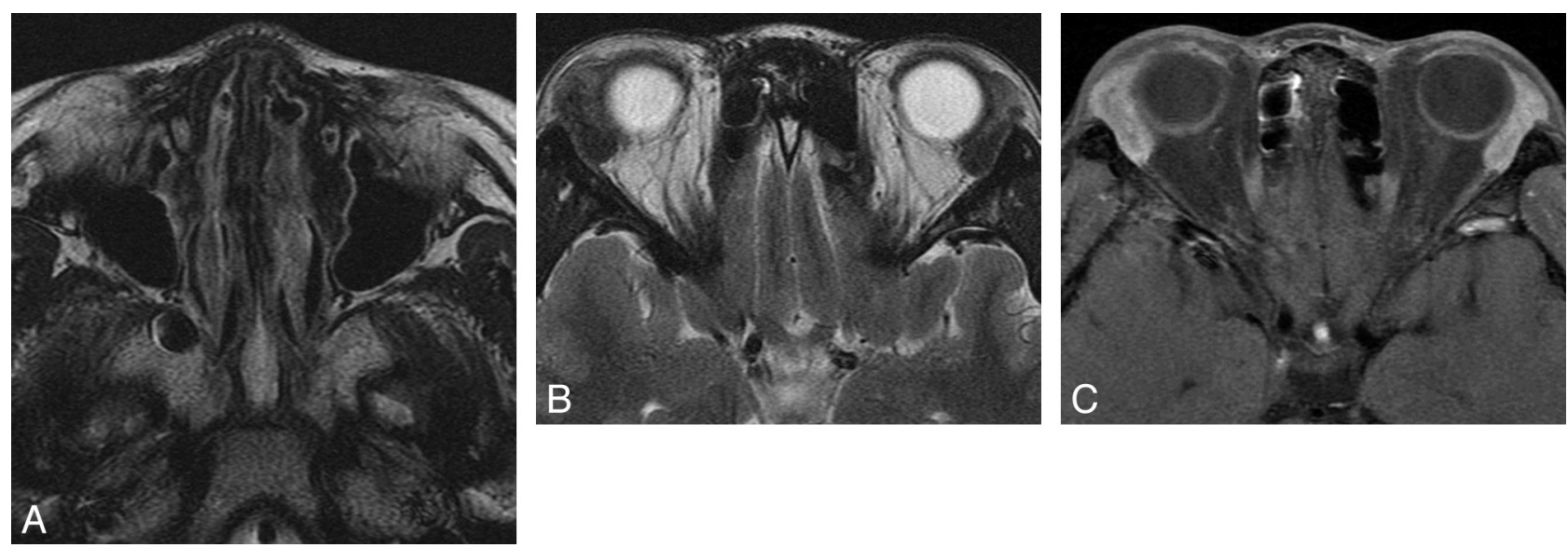

Fig 4. Case 2. A, Axial T2-weighted image shows diffuse thickening of the septum and lateral nasal walls with hypointense signal intensity. $B$, Axial T2-weighted image shows bilateral lacrimal gland enlargement, appearing as hypointense signal intensity. C, Axial contrast-enhanced T1-weighted image with fat saturation shows moderate homogeneous enhancement of bilateral lacrimal glands.

nasal cavity, with necrosis, facial subcutaneous or nasolabial fold soft-tissue infiltration, midline destruction, and extension into the nasopharynx. ${ }^{23}$ Inverted papilloma frequently originate from the lateral nasal wall, appearing lobulate in shape. Most lesions typically show a convoluted cerebriform pattern on both T2-weighted and contrast-enhanced T1weighted MR images. ${ }^{24}$ Polyps are characterized by bilateral involvement and most frequently arise in the middle meatus, the ethmoid infundibulum, and the uncinate process. Polyps usually show low signal intensity on T1-weighted MR images and hyperintense signal intensity on T2-weighted images, without enhancement of internal contents following the administration of contrast material. Hemangioma reveals obviously high signal intensity on T2-weighted MR images, with marked contrast enhancement. Schwannoma seldom occurs in the nasal cavity. It typically has cystic areas, due to the Antoni B component, which exhibits high signal intensity on T2weighted images and has no enhancement on contrast-enhanced T1-weighted images.

Surgical excision appears to be the treatment of choice, though recurrences are common in most reported cases and multiple excisions are frequently required. Steroid therapy has been tried in some cases but usually produces poor results. $^{2,5,10-14,19}$

\section{Conclusions}

Thorough knowledge of the typical CT and MR imaging features of EAF, combined with history, can help to identify the diagnosis. CT and MR imaging are complementary techniques in evaluating the extent of the lesions and adjacent bony changes before deciding on a strategy for therapy. Although EAF is extremely rare in the nasal region, it should be considered in the differential diagnosis of nasal lesions.

\section{References}

1. Roberts PF, McCann BG. Eosinophilic angiocentric fibrosis of the upper respiratory tract: a mucosal variant of granuloma faciale? A report of three cases. Histopathlogy 1985;9:1217-25

2. Fageeh NA, Mai KT, Odell PF. Eosinophilic angiocentric fibrosis of the subglottic region of the larynx and upper trachea. J Otolaryngol 1996;25:276-78
3. Roberts PF, McCann BG. Eosinophilic angiocentric fibrosis of the upper respiratory tract: a postscript. Histopathology 1997;31:385-86

4. Matai V, Baer S, Barnes S, et al. Eosinophilic angiocentric fibrosis. J Laryngol Otol 2000;114:563-64

5. Thompson LD, Heffner DK. Sinonasal tract eosinophilic angiocentric fibrosis. A report of three cases. Am J Clin Pathol 2001;115:243-48

6. Owa AO, Boyle S, Gallimore AP. Eosinophilic angiocentric fibrosis as a cause of nasal obstruction. Rhinology 2001;40:41-43

7. Burns BV, Roberts PF, De Carpentier J, et al. Eosinophilic angiocentric fibrosis affecting the nasal cavity. A mucosal variant of the skin lesion granuloma faciale. J Laryngol Otol 2001;115:223-26

8. Pereira EM, Millas I, Reis-Filho JS, et al. Eosinophilic angiocentric fibrosis of the sinonasal tract: report on the clinicopathologic features of a case and review of the literature. Head Neck 2002;24:307-11

9. Tabee A, Zadeh MH, Proytcheva M, et al. Eosinophilic angiocentric fibrosis. J Laryngol Otol 2003;117:410-13

10. Goldman NC. Angiocentric eosinophilic fibrosis. Otolaryngol Head Neck Surg 2003;128:445-46

11. Nguyen DB, Alex JC, Calhoun B. Eosinophilic angiocentric fibrosis in a patient with nasal obstruction. Ear Nose Throat J 2004;83:183-84,186

12. Narayan J, Douglas-Jones AG. Eosinophilic angiocentric fibrosis and granuloma faciale: analysis of cellular infiltrate and review of the literature. Ann Otol Rhinol Laryngol 2005;114:35-42

13. Paun S, Lund VJ, Gallimore AP. Nasal fibrosis: long-term follow up of four cases of eosinophilic angiocentric fibrosis. J Laryngol Otol 2005;119: $119-24$

14. Slovik Y, Putterman M, Nash M, et al. Eosinophilic angiocentric fibrosis of the sinonasal tract in a male patient with chronic bowel inflammation. Am J Rhinol 2006;20:91-94

15. Valenzuela AA, Whitehead KJ, Brown I, et al. Eosinophilic angiocentric fibrosis: an unusual entity producing complete lacrimal duct obstruction. Orbit 2006;25:159-61

16. Leibovitch I, James CL, Wormald PJ, et al. Orbital eosinophilic angiocentric fibrosis case report and review of the literature. Ophthalmology 2006;113: $148-52$

17. Onder S, Sungur A. Eosinophilic angiocentric fibrosis: an unusual entity of the sinonasal tract. Arch Pathol Lab Med 2004;128:90-91

18. Kiratli H, Onder S, Yildiz S, et al. Eosinophilic angiocentric fibrosis of the orbit. Clin Experiment Opthalmol, 2008;36:274-76

19. Clauser L, Mandrioli S, Polito J, et al. Eosinophilic angiocentric fibrosis. J Craniofac Surg 2006;17:812-14

20. Kosarac O, Luna MA, Ro JY, et al. Eosinophilic angiocentric fibrosis of the sinonasal tract. Ann Diagn Pathol 2008;12:267-70

21. Yabuuchi H, Fukuya T, Tajima T, et al. Salivary gland tumors: diagnostic value of gadolinium-enhanced dynamic MR imaging with histopathologic correlation. Radiology 2003;226:345-54

22. Westreich RW, Lawson W. Middle necrotizing nasal lesions: analysis of 18 cases emphasizing radiological and serological findings with algorithms for diagnosis and management. Am J Rhinol 2004;18:209-19

23. King AD, Lei KI, Ahuja AT, et al. MR imaging of nasal T-cell / natural killer cell lymphoma. AJR Am J Roentgenol 2000;174:209-11

24. Ojiri H, Ujita M, Tada S, et al. Potentially distinctive features of sinonasal inverted papilloma on MR imaging. AJR Am J Roentgenol 2000;175:465-68 